Research Paper

\title{
Prognostic Significance of Hematological Markers for Patients with Nasopharyngeal Carcinoma: A Meta-analysis
}

\author{
Shanshan Yang"\#, Kun Zhao#, Xiao Ding1, Haiping Jiang1, Haijun Lu1 ${ }^{1 凶}$ \\ 1. Department of Oncology, the Affiliated Hospital of Qingdao University, Qingdao, Shandong Province, China \\ 2. Key Laboratory of Tissue Engineering and Regenerative Medicine of Zhejiang Province, School of Medicine, Zhejiang University, Hangzhou, Zhejiang \\ Province, China \\ \#Equal contributors (Co-first authors) \\ $\triangle$ Corresponding author: Haijun Lu. E-mail: M201576106@hust.edu.cn; Tel.: +86-0532-82911348 \\ (C) Ivyspring International Publisher. This is an open access article distributed under the terms of the Creative Commons Attribution (CC BY-NC) license \\ (https://creativecommons.org/licenses/by-nc/4.0/). See http://ivyspring.com/terms for full terms and conditions.
}

Received: 2018.04.19; Accepted: 2019.04.02; Published: 2019.06.02

\begin{abstract}
Purpose: Hematological parameters are considered to be associated with prognosis in various cancers. We designed a meta-analysis to evaluate the prognostic significance of hematological parameters, including the neutrophil-to-lymphocyte ratio (NLR), C-reactive protein/albumin ratio (CRP/ALB), lymphocyte-to-monocyte ratio (LMR), plasma fibrinogen level, Glasgow prognostic score (GPS), platelet-to-lymphocyte ratio (PLR) and high-density lipoprotein cholesterol (HDL-C) level, on clinical outcomes in nasopharyngeal carcinoma (NPC).

Methods: Relevant studies published prior to February 2018 were identified in the PubMed, Web of Science, EMBASE and Cochrane library databases. The primary outcome was overall survival (OS), and the secondary outcome was progression-free survival (PFS). The pooled hazard ratios (HRs) and $95 \%$ confidence intervals $(\mathrm{Cls})$ were calculated.

Results: In total, 23 studies encompassing 23,417 patients were included in our meta-analysis. An elevated NLR was related to a poor OS (HR=1.46, 95\% Cl=1.30-1.63, $p<0.00001)$ and PFS $(\mathrm{HR}=1.67,95 \% \mathrm{Cl}=1.36-2.07, p<0.00001)$, and a high PLR was associated with a poor OS $(\mathrm{HR}=1.62$, $95 \% \mathrm{Cl}=1.32-1.98, p<0.00001)$. Additionally, a high LMR predicted a significantly favorable OS $(\mathrm{HR}=0.50,95 \% \mathrm{Cl}: 0.43-0.58, p<0.00001)$. CRP/ALB, the GPS, HDL-C and plasma fibrinogen levels were also related to OS and PFS.

Conclusion: Inflammation-based prognostic scoring systems considering inflammatory cells (lymphocytes, neutrophils, platelets and monocytes) and proteins (ALB, CRP and HDL-C) are essential prognostic factors.
\end{abstract}

Key words: nasopharyngeal carcinoma; hematological markers; inflammatory cells and proteins; overall survival

\section{Introduction}

Nasopharyngeal carcinoma (NPC) is a squamous-cell cancer that has a remarkable worldwide ethnic and geographic distribution $[1,2]$. High incidence rates of 20-30 per 100,000 people have been reported in South China, especially in Guangdong Province, and these rates are 100-fold higher than those in Western countries [3]. Because of its anatomic location and radiosensitivity, radiotherapy, especially intensity modulated radiotherapy (IMRT), has become the standard treatment for NPC, and recently, the value of targeted therapy is increasingly appreciated [4]. Although early-stage NPC can be cured, patients usually present with advanced NPC at primary diagnosis, and $20-30 \%$ of patients develop local recurrence and/or distant metastasis [5]. The TNM 
staging system is the gold standard for predicting NPC prognosis; however, this system cannot reflect the biological heterogeneity among tumors. Thus, the identification of reliable biomarkers is highly important for improving prognosis prediction and complementing the existing TNM staging system [6, 7]. Inflammatory cells and proteins play a critical role in tumor development and may serve as prognostic factors in various cancers. Indeed, correlations between prognosis and several hematological biomarkers, such as the lymphocyte ratio (NLR), lymphocyte-to-monocyte ratio (LMR) and Glasgow prognostic score (GPS), have been identified in a wide range of cancers [8-13]. Furthermore, plasma fibrinogen levels and high-density lipoprotein cholesterol (HDL-C) levels have been reported to have prognostic value in predicting survival in colon cancer and gastric cancer, respectively [14]. These markers are more easily available and less expensive to assess than other reported markers. Nevertheless, the prognostic significance of these hematological biomarkers in NPC has not been fully elucidated, and further studies are needed. Accordingly, we conducted a meta-analysis to measure the ability of the abovementioned hematological biomarkers to predict clinical outcomes in NPC.

\section{Methods}

\section{Search strategy}

We referred to the Preferred Reporting Items for Systematic Reviews and Meta-Analyses (PRISMA) statement in our analysis [15]. A systematic literature search was performed to identify articles published prior to February 2018 in the PubMed, Web of Science, EMBASE and Cochrane library. The search terms were as follows: "hematologic markers", "NLR" ("neutrophil-to-lymphocyte ratio", "neutrophil lymphocyte ratio", "neutrophil to lymphocyte ratio", and "neutrophil-lymphocyte ratio"), "LMR" ("lymphocyte-monocyte ratio", "lymphocyte to monocyte ratio", "lymphocyte monocyte ratio", and "lymphocyte-to-monocyte ratio), "HDL-C" ("highdensity lipoprotein cholesterol" and "alpha-Lipoproteins"), "PLR" ("platelet lymphocyte ratio", "platelet to lymphocyte ratio", "platelet-tolymphocyte ratio", and "platelet-lymphocyte ratio"), "GPS" ("Glasgow Outcome Scale" and "Glasgow prognostic score"), "CRP to ALB" or "C-reactive Protein to Albumin Ratio", "plasma fibrinogen", "prognosis" ("prognosis", "outcome", "survival", and "mortality"), and "NPC" ("Nasopharyngeal Neoplasms" and "Nasopharyngeal Cancer"). In addition, references from the identified publications were also retrieved.

\section{Inclusion and exclusion criteria}

The inclusion criteria: (1) NPC diagnosed based on pathological examination; (2) studies including an evaluation of the prognosis value of at least 1 hematologic biomarker, such as NLR, PLR, LMR, CRP/ALB, GPS, plasma fibrinogen or HDL-C, in NPC; and (3) HRs and 95\% CIs or p-values available or possible to calculate.

The exclusion criteria: (1) reviews, letters, laboratory studies and case reports; (2) studies not written in English.

\section{Data extraction and quality assessment}

The data were extracted from the qualified studies by two investigators (SSY and KZ) independently. The following information was recorded: country, year, author's name, sample size, survival outcomes, cut-off value, follow-up, disease stage, HRs, and $95 \% \mathrm{CIs}$ or p-values of overall survival (OS) and progression-free survival (PFS). If available, we preferentially extracted the HRs from multivariable analyses; otherwise, the HRs were extracted from univariate analyses. The quality of the eligible studies was evaluated by the Newcastle-Ottawa Quality Scale (NOS), which includes the following aspects: selection (four points), outcome assessment (three points) and comparability (two points). Studies with scores $\geq 6$ were considered high quality.

\section{Statistical analyses}

The impact of the hematological biomarkers on OS and PFS was evaluated by pooled HRs and corresponding $95 \%$ CIs, which were directly obtained from the studies or calculated according to the methods presented by Tierney [16]. Heterogeneity was measured by Cochrane $Q$ test and the $\mathrm{I}^{2}$ statistic. A random-effects model was selected if there was significant heterogeneity $\left(p<0.05\right.$ and $\left.\mathrm{I}^{2}>50 \%\right)$; otherwise, a fixed-effects model was used. We performed a sensitivity analysis by excluding a study and recalculating the combined HRs. Publication bias was evaluated by Begg's funnel plots and Egger's tests. The statistical analyses were conducted using RevMan5.3 (Cochrane Collaboration) and $p<0.05$ was considered statistically significant.

\section{Results}

\section{Search results and study characteristics}

The flow chart of literature retrieval is presented in Figure 1. In total, 152 studies were initially retrieved from the electronic databases; of these studies, 53 were duplicates. Thirty-five articles remained after screening the titles or abstracts, and 
then, 12 articles were excluded due to the lack of adequate data. Thus, 23 studies involving 23,417 patients were included in our meta-analysis; the sample sizes ranged from 108 to 3,237. Except for one study, all studies collected data retrospectively [17]. The studies were conducted in Asia and published after 2011. The mean score of NOS is 6.95 with a range from 6 to 8 . The characteristics of the eligible studies are displayed in Table 1.

\section{Correlation between hematologic parameters and survival outcomes}

\section{Impact of NLR on OS and PFS in NPC}

The association between NLR and OS was illustrated in 10 studies involving 7,031 NPC patients. In all studies, a high NLR was related to a poor OS with a pooled $\mathrm{HR}$ of $1.46 \quad(95 \% \mathrm{CI}=1.30-1.63$, $p<0.00001)$. Minimal heterogeneity was observed $\left(\mathrm{I}^{2}=9 \%\right)$, as illustrated by the forest plot displayed in Figure 2. The cut-off values for NLR ranged from 2.28 to 5.0. In total, there were 2,012 patients from 4 studies in the NLR analysis of PFS. As depicted in Figure 3a, an NLR higher than the cut-off was related to a poor PFS (HR=1.67, 95\% CI=1.36-2.07, $p<0.00001)$ in NPC without heterogeneity $\left(\mathrm{I}^{2}=0 \%\right)$.

\section{Prognostic effect of elevated PLR on OS}

Five studies involving 2,520 participants described the effect of PLR on the NPC prognosis. The combined analysis revealed that a high PLR was related to a poor OS $(\mathrm{HR}=1.62,95 \% \mathrm{CI}=1.32-1.98$, $p<0.00001)$ without significant heterogeneity $\left(\mathrm{I}^{2}=20 \%\right)$ (Figure 2).

Table 1: Characteristics of the included studies

\begin{tabular}{|c|c|c|c|c|c|c|c|c|c|}
\hline Author (year) & Nos. & Country & $\begin{array}{l}\text { Prognostic } \\
\text { makers }\end{array}$ & Cut-off & Study type & $\begin{array}{l}\text { No. of } \\
\text { patients }\end{array}$ & HR & Survival outcome & $\begin{array}{l}\text { Follow-up (months)/median } \\
\text { (range) }\end{array}$ \\
\hline Cui Chen 2014 [56] & 7 & China & $\begin{array}{l}\text { NLR } \\
\text { PLR } \\
\text { GPS }\end{array}$ & $\begin{array}{l}5 \\
150 \\
1\end{array}$ & retrospective & 211 & $\mathrm{U} / \mathrm{M}$ & PFS/OS & NA \\
\hline Jian-Rong He 2012 [57] & 8 & China & NLR & 2.74 & retrospective & 1410 & $\mathrm{U} / \mathrm{M}$ & PFS/OS & $41(2-60)$ \\
\hline Wei Sun 2016 [58] & 8 & China & $\begin{array}{l}\text { NLR } \\
\text { PLR }\end{array}$ & $\begin{array}{l}2.6 \\
163.4\end{array}$ & retrospective & 251 & $\mathrm{U} / \mathrm{M}$ & PFS/OS & $50(5-84)$ \\
\hline Rou Jiang 2015 [59] & 7 & China & LMR & 2.475 & retrospective & 672 & $\mathrm{U} / \mathrm{M}$ & OS & NA \\
\hline Rou Jiang 2015 [60] & 7 & China & PLR & 153.64 & retrospective & 1261 & $\mathrm{U} / \mathrm{M}$ & $\begin{array}{l}\text { OS/CSS/ } \\
\text { DMFS }\end{array}$ & NA \\
\hline $\begin{array}{l}\text { Ying Jin } \\
2015 \text { [61] }\end{array}$ & 7 & China & NLR & 3.6 & retrospective & 229 & $\mathrm{U} / \mathrm{M}$ & OS & NA \\
\hline $\begin{array}{l}\text { Jing Li } \\
2013[62]\end{array}$ & 7 & China & LMR & 5.22 & retrospective & 1547 & $\mathrm{U} / \mathrm{M}$ & $\begin{array}{l}\text { OS/DFS/ } \\
\text { DMFS }\end{array}$ & $67.07(1.41-99.02)$ \\
\hline Xiao-Hui Li 2016 [63] & 6 & China & $\begin{array}{l}\text { NLR } \\
\text { PLR } \\
\text { LMR } \\
\text { GPS }\end{array}$ & $\begin{array}{l}2.5 \\
166 \\
2.35 \\
1\end{array}$ & retrospective & 388 & $\mathrm{U} / \mathrm{M}$ & DSS & NA \\
\hline Gui-Nan Lin 2014 [64] & 7 & China & LMR & 5.07 & retrospective & 256 & $\mathrm{U} / \mathrm{M}$ & OS & $22.6(5.1-42.3)$ \\
\hline Jian-Pei Li 2016 [65] & 6 & China & $\begin{array}{l}\text { NLR } \\
\text { PLR } \\
\text { GPS } \\
\text { CRP/ALB }\end{array}$ & $\begin{array}{l}2.48 \\
146.2 \\
1 \\
0.03\end{array}$ & retrospective & 409 & $\mathrm{U} / \mathrm{M}$ & OS & NA \\
\hline Xin An 2011 [66] & 7 & China & NLR & 3.73 & retrospective & 363 & M & DSS/DMFS/LRFS & $62(2-92)$ \\
\hline H. Chang 2013 [67] & 7 & China & NLR & 2.5 & retrospective & 3237 & M & DSS & NA \\
\hline $\begin{array}{l}\text { Melvin Lee Kiang Chua } 2016 \\
\text { [68] }\end{array}$ & 8 & Singapore & NLR & 3 & prospective & 393 & $\mathrm{U} / \mathrm{M}$ & $\begin{array}{l}\text { OS/DFS/ } \\
\text { DMFS }\end{array}$ & NA \\
\hline Chua 2015 [69] & $\mathrm{NA}^{*}$ & Singapore & NLR & 3 & $\mathrm{RCT}$ & 108 & $\mathrm{U}$ & DFS/DFMS & $27.6(1.32-47.04)$ \\
\hline Aiying Lu 2017 [70] & 8 & China & NLR & 2.28 & retrospective & 140 & M & PFS/OS & $68(5-77)$ \\
\hline Chang-Juan Tao 2016 [71] & 7 & China & CRP/ALB & 0.141 & retrospective & 719 & $\mathrm{U} / \mathrm{M}$ & OS/DMFS & $48(3-89)$ \\
\hline ShaSha He 2016 [72] & 7 & China & CRP/ALB & 0.064 & retrospective & 2685 & $\mathrm{U} / \mathrm{M}$ & OS & $46.30(0.07-188.43)$ \\
\hline Yuan Zhang 2017 [73] & 6 & China & CRP/ALB & 0.05 & retrospective & 1572 & M & $\begin{array}{l}\text { OS/DFS/ } \\
\text { DMFS }\end{array}$ & NA \\
\hline Yan-Yan Liu 2016 [74] & 6 & China & HDL-C & 1.295 & retrospective & 2443 & M & OS & $95.2(51.6-110.6)$ \\
\hline $\begin{array}{l}\text { Rou Jiang } \\
2014 \text { [75] }\end{array}$ & 7 & China & HDL-C & 0.965 & retrospective & 807 & M & OS & NA \\
\hline $\begin{array}{l}\text { Sha-ShaHeB } \\
2017 \text { [76] }\end{array}$ & 6 & China & Fibrinogen & 3.345 & retrospective & 998 & M & OS/DMFS & NA \\
\hline Mei Lan 2016 [18] & 7 & China & Fibrinogen & 4 & retrospective & 755 & M & $\begin{array}{l}\text { DSS/DFS } \\
\text { /DMFS }\end{array}$ & $50(3-87)$ \\
\hline L-Q Tang 2014 [77] & 7 & China & Fibrinogen & 3.34 & retrospective & 2563 & $\mathrm{U}$ & $\begin{array}{l}\text { OS/DFS/ } \\
\text { DMFS }\end{array}$ & NA \\
\hline
\end{tabular}

NA*: Newcastle-Ottawa Quality Scale (NOS) is not suitable for RCT.

Abbreviations: HR: hazard ratio; NA: not available; NLR: neutrophil-to-lymphocyte ratio; PLR: platelet-to-lymphocyte ratio; LMR: lymphocyte-to-monocyte ratio; CRP/ALB: C-reactive protein/albumin ratio; GPS: Glasgow prognostic score; HDL-C: high-density lipoprotein cholesterol; OS: overall survival; PFS: progression-free survival; PFS: disease-free survival; DMFS: distant metastasis-free survival. 


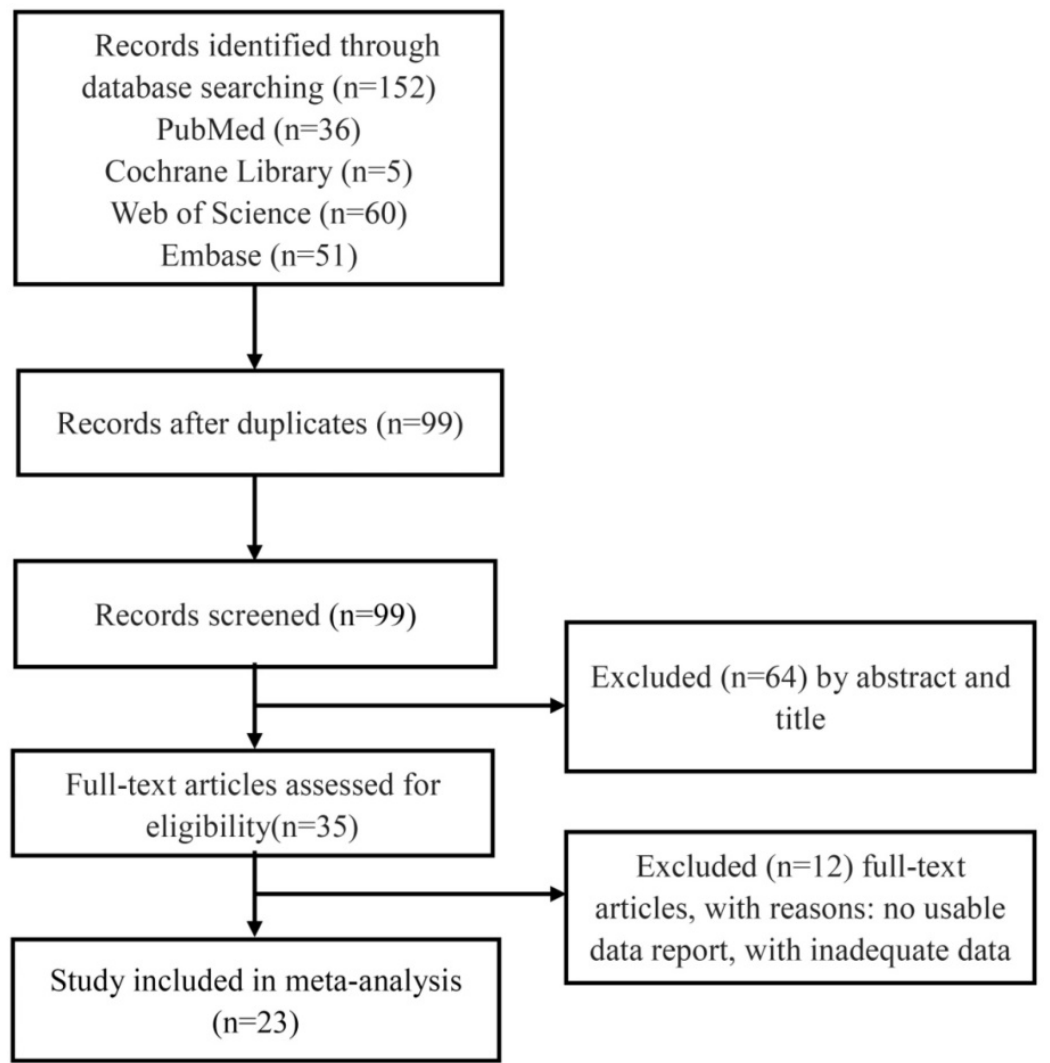

Figure 1: Flow chart of the included studies in this meta-analysis.

Prognostic effect of LMR on OS

Four studies involving 2,863 participants reported the effect of LMR on OS. The combined data showed that a high LMR was related to a favorable OS (HR=0.50, 95\% CI: 0.43-0.58, $p<0.00001)$ without heterogeneity $\left(\mathrm{I}^{2}=0 \%\right)$ (Figure 2$)$.

\section{Prognostic effect of CRP/ALB on OS}

The effect of CRP/ALB on OS was reported in 4 studies involving 5,385 patients. Compared with a low CRP/ALB, a high CRP/ALB was obviously related to a poor OS $(\mathrm{HR}=1.48,95 \% \mathrm{CI}$ : 1.27-1.72, $p<0.00001$; Figure 2) without significant heterogeneity $\left(\mathrm{I}^{2}=31 \%\right)$.

\section{Prognostic effect of GPS on OS}

Three studies presenting data regarding the effect of GPS on OS among 1008 patients were found. The pooled analysis indicated that a high GPS was obviously related to a poor OS ( $\mathrm{HR}=2.53,95 \% \mathrm{CI}$ : $2.02-3.18, p<0.00001$; Figure 2), and no heterogeneity $\left(\mathrm{I}^{2}=0 \%\right)$ was observed.

\section{Prognostic effect of HDL-C on OS}

Only two studies reported the effects of the HDL-C levels on OS, and the pooled analysis showed that HDL-C was not related to OS (HR=1.22, 95\% CI: $0.52-2.86, p=0.65$; Figure $3 b$ ).

Prognostic significance of plasma fibrinogen on OS, DFS and DMFS

The effect of plasma fibrinogen on OS, DFS and DMFS was described in 2 studies, 2 studies, and 3 studies, respectively. The pooled HR value suggested that an elevated level of plasma fibrinogen predicted a poor OS (HR=1.90, 95\% CI: 1.46-2.49, $p<0.00001$, Figure 2) and DFS (HR=1.59, 95\% CI: 1.32-1.92, $p<0.00001$, Figure 3c) with no heterogeneity $\left(\mathrm{I}^{2}=0 \%\right)$; the same effect was found on DMFS (HR=1.96, 95\% CI: 1.32-2.91, $p=0.008)$ but with significant heterogeneity $\left(\mathrm{I}^{2}=79 \%\right)$ (Figure $\left.4 \mathrm{a}\right)$.

\section{Sensitivity analyses}

Regarding the pooled HR of the effect of plasma fibrinogen on DMFS, the sensitivity analysis showed that one particular study conducted by Mei Lan et al. [18] markedly affected the pooled effects as the combined HR changed from 1.96 (95\% CI: 1.32-2.91, $p=0.008, \mathrm{I}^{2}=79 \%$ ) to 1.59 (95\% CI: $1.31-1.94 ; p<0.00001$, $\mathrm{I}^{2}=0 \%$ ) when this study was omitted (Figure $4 \mathrm{~b}$ ). We also performed the sensitivity analysis by individually omitting other studies, although no single study substantially affected the pooled outcome. 


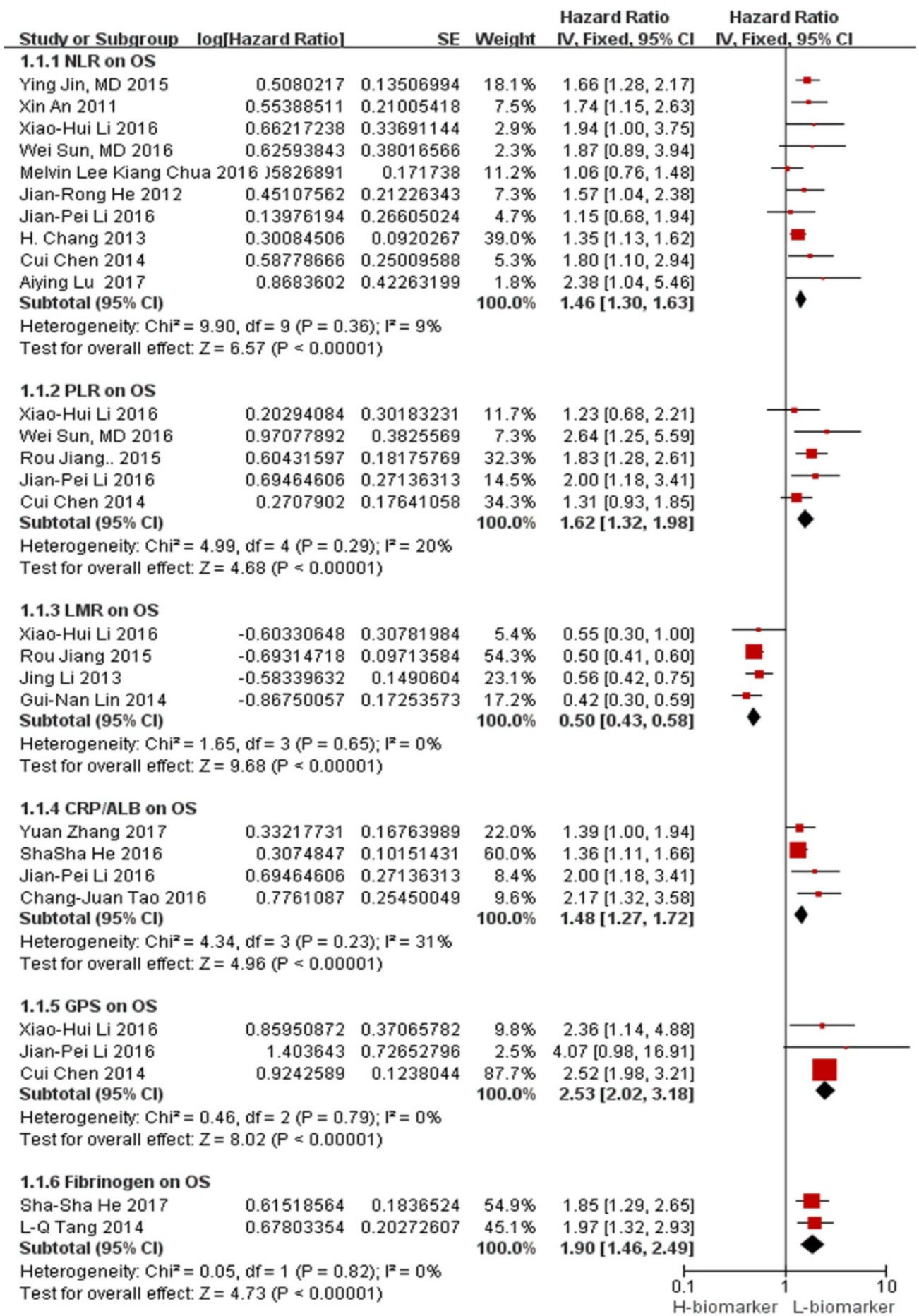

Figure 2: Forest plots of the hematological parameters for OS. Abbreviations: NLR: neutrophil-to-lymphocyte ratio; PLR: platelet-to-lymphocyte ratio; LMR: lymphocyte-to-monocyte ratio; CRP/ALB: C-reactive protein/albumin ratio; GPS: Glasgow prognostic score; OS: overall survival.

\section{Publication bias}

We used Begg's funnel plots and Egger's tests to screen for potential publication bias, and no clear publication bias was observed.

\section{Discussion}

To date, various markers, including hematological parameters, have been applied for the prediction of tumor outcomes. Increasing evidence reveals that a relationship exists between the systematic inflammatory response and carcinoma, and the association with inflammatory proteins (GPS, plasma fibrinogen and HDL-C) has also been reported. These markers can be inexpensive and are readily available. Thus, we performed this meta-analysis to evaluate the prognostic significance of hematological parameters in NPC. 


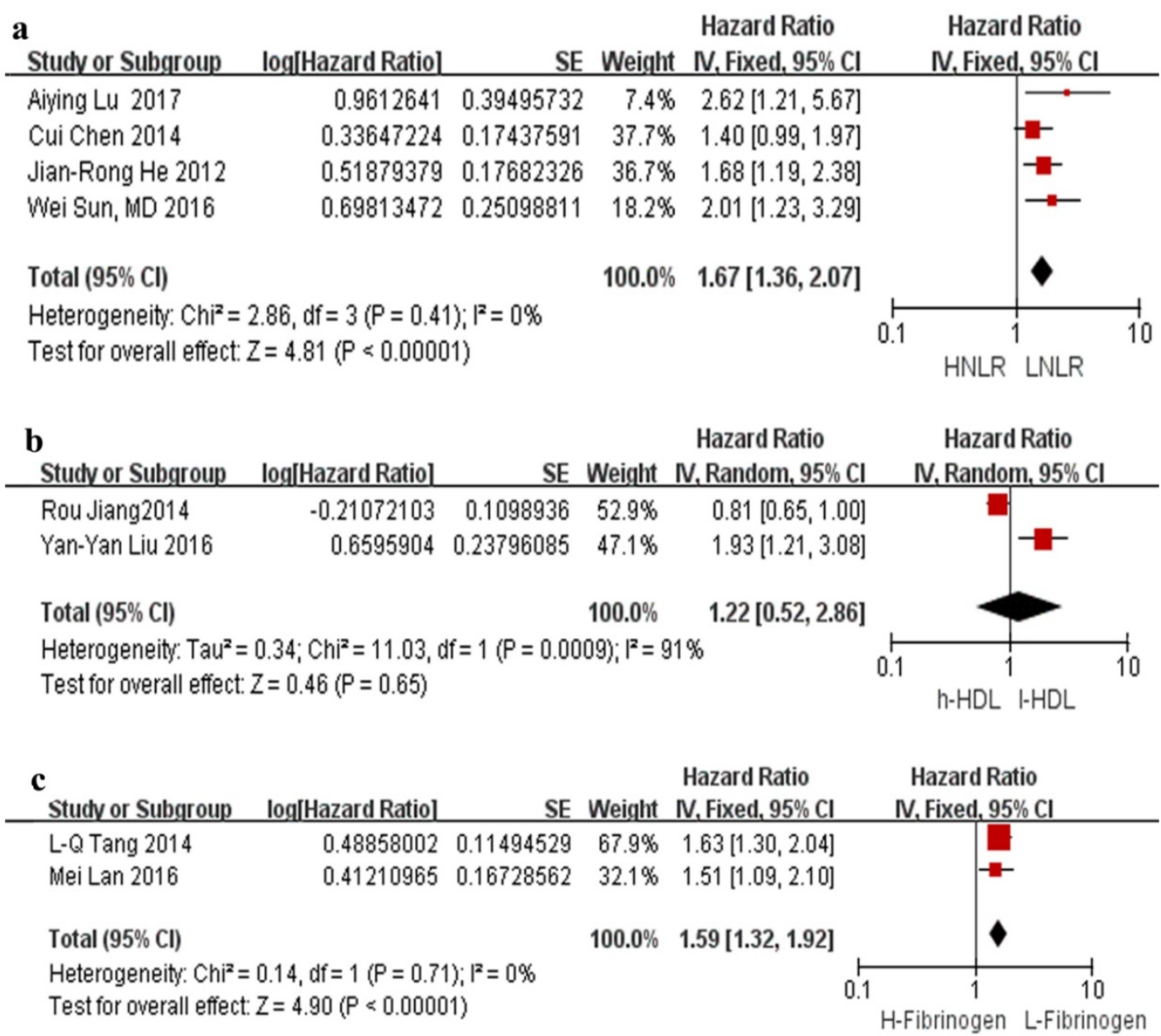

Figure 3: Forest plots of NLR for PFS (a), HDL for OS (b), and fibrinogen for DFS (c). Abbreviations: HNLR: high neutrophil-to-lymphocyte ratio; LNLR: low neutrophil-to-lymphocyte ratio; PFS: progression-free survival; h-HDL: high high-density lipoprotein cholesterol; I-HDL: low high-density lipoprotein cholesterol; H-Fibrinogen: high fibrinogen; L-Fibrinogen: low fibrinogen; DFS: disease-free survival.

\begin{tabular}{|c|c|c|c|c|c|}
\hline${ }^{\mathbf{a}}$ Study or Subgroup & log[Hazard Ratio] & SE & Weight & $\begin{array}{c}\text { Hazard Ratio } \\
\text { IV, Random, } 95 \% \mathrm{Cl}\end{array}$ & $\begin{array}{c}\text { Hazard Ratio } \\
\text { IV. Random, } 95 \% \mathrm{Cl}\end{array}$ \\
\hline L-Q Tang 2014 & 0.51879379 & 0.14361854 & $34.8 \%$ & $1.68[1.27,2.23]$ & $\mid$\begin{tabular}{l|l}
- \\
\end{tabular} \\
\hline Mei Lan 2016 & 1.15373159 & 0.20015989 & $29.8 \%$ & $3.17[2.14,4.69]$ & $\rightarrow$ \\
\hline Sha-Sha He 2017 & 0.41739368 & 0.13777409 & $35.3 \%$ & $1.52[1.16,1.99]$ & $\Rightarrow$ \\
\hline Total $(95 \% \mathrm{Cl})$ & & & $100.0 \%$ & $1.96[1.32,2.91]$ & \\
\hline $\begin{array}{l}\text { Heterogeneity: } \mathrm{Tau}^{2}= \\
\text { Test for overall effect: }\end{array}$ & $\begin{array}{l}0.10 ; C^{2}=9.73, d f \\
Z=3.34(P=0.0008\end{array}$ & $=2(P=0.008$ & $; 1^{2}=79 x$ & & $\begin{array}{ccc}1 & 1 & 10 \\
\text { H-Fibrinogen } & \text { L-Fibrinogen }\end{array}$ \\
\hline b $_{\text {Study or Subgroup }}$ & log[Hazard Ratio] & SE & Weight & $\begin{array}{l}\text { Hazard Ratio } \\
\text { IV, Fixed, } 95 \% \mathrm{Cl}\end{array}$ & $\begin{array}{c}\text { Hazard Ratio } \\
\text { IV, Fixed, } 95 \% \mathrm{Cl}\end{array}$ \\
\hline L-Q Tang 2014 & 0.51879379 & 0.14361854 & $47.9 \%$ & $1.68[1.27,2.23]$ & \\
\hline Sha-Sha He 2017 & 0.41739368 & 0.13777409 & $52.1 \%$ & $1.52[1.16,199]$ & \\
\hline Total $(95 \% \mathrm{Cl})$ & & & $100.0 \%$ & $1.59[1.31,1.94]$ & \\
\hline $\begin{array}{l}\text { Heterogeneity: } \mathrm{Chi}^{2}= \\
\text { Test for overall effect }\end{array}$ & $\begin{array}{l}0.26, d f=1(P=0.6 \\
Z=4.69(P<0.0000\end{array}$ & $\begin{array}{l}\text { 1); }\left.\right|^{2}=0 \% \\
\text { 1) }\end{array}$ & & $\begin{array}{r}0.1 \\
H-\end{array}$ & ${ }^{1}$ brinogen 10 \\
\hline
\end{tabular}

Figure 4: Forest plots of the pooled analysis of the effects of fibrinogen on DMFS (a) and sensitivity analysis of the effect of fibrinogen on DMFS (b). Abbreviations: H-Fibrinogen: high fibrinogen; L-Fibrinogen: low fibrinogen; DMFS: distant metastasis-free survival.

Our pooled analysis showed that hematological markers, including NLR, PLR, LMR, CRP/ALB, GPS and plasma fibrinogen, are related to NPC survival outcomes in Asian populations. Patients with a high
LMR were deemed to have a favorable prognosis, whereas high NLR, PLR, CRP/ALB, GPS and plasma fibrinogen were related to a poor prognosis. In addition, the pooled analysis revealed that HDL-C 
was not associated with prognosis; however, as only two studies were eligible for our analysis, further studies are required.

Although the exact mechanism between hematological parameters and malignancies has not been reported, this association may be illustrated by tumor-infiltrating immune cells and inflammatory proteins. Lymphocytes play a crucial role in the immunologic antitumor responses by inhibiting tumor cell proliferation and inducing cell death. In fact, tumor-infiltrating lymphocytes (TILs) are related to a good prognosis in various cancers [19, 20]. Moreover, by inducing apoptosis in tumor cells, the interaction between $\mathrm{CD} 8+$ and $\mathrm{CD} \mathrm{4+} \mathrm{T}$ cells is necessary for the antitumor reaction[21, 22], and increased infiltration of CD8+ $\mathrm{T}$ lymphocytes has been reported to improve OS and PFS in NPC[23]. Overall, effective immunotherapy for NPC may depend on the ability to generate cytotoxic $\mathrm{T}$ lymphocytes (CTLs) that can home to nasopharyngeal tissue and other metastatic sites. Nevertheless, platelets may protect tumor cells from immune elimination, support tumor metastasis [24] and mediate tumor cell growth, angiogenesis and dissemination by secreting a variety of growth factors [25-27]. Furthermore, an elevated platelet count is reportedly related to a poor prognosis in cancers [28, 29]. Moreover, neutrophils, representing another type of inflammatory cells, may contribute to tumor cell growth, angiogenesis and metastasis by producing cytokines and releasing angiogenic factors [30]. In addition, macrophages derived from circulating monocytes might accelerate tumor progression and angiogenesis [31]. Increasing evidence shows that tumor-associated macrophages (TAMs) are related to worse OS [32], and Bingle et al. [33] demonstrated that the macrophage density in tumors is related to a poor prognosis. In accordance with the above hypothesis, our study determined that high PLR and NLR values are related to poor OS and PFS, whereas high LMR is associated with a favorable prognosis. CRP, which is an acute-phase response protein, is mainly synthesized by hepatocytes induced by pro-inflammatory cytokines [10]. Elevated CRP is related to inflammation accompanied by hypoalbuminemia, which results in dystrophia and further leads to a poor prognosis. The CRP/ALB ratio reveals the relationship between systemic inflammatory responses and malnutrition and has been reported to have a prognostic effect in ovarian cancer [34], hepatocellular carcinoma [10], pancreatic cancer [35], lung cancer [36] and esophageal carcinoma [37]. In our analysis, we combined 4 studies involving 5,385 patients and found that a high CRP/ALB ratio was a significant predictor of an inferior OS in NPC. GPS is another well-recognized inflammation-based prognostic score demonstrated to be associated with survival in various cancers, such as intrahepatic cholangiocarcinoma and ovarian cancer [38-40]. Consistent with the above results, our study indicated that a high GPS was obviously related to poor OS with a convincing conclusion. Additionally, fibrinogen, which is an acute-phase glycoprotein and an important clotting factor, is converted to insoluble fibrin by thrombin, which affects the inflammatory response. Fibrinogen is a bridging factor promoting endothelial adhesion in target organs [41, 42]. Fibrinogen has also been proven to be a prognostic marker in several cancers, such as ovarian cancer and hepatocellular carcinoma [43]. Furthermore, a nomogram based on fibrinogen was generated to predict its prognostic significance in lung cancer, and high fibrinogen levels were related to a shorter OS [44]. In our study, high serum fibrinogen levels were associated with a poor NPC patient prognosis. Moreover, HDL-C, which is a key lipoprotein in cholesterol transport involved in certain signaling pathways important for malignant transformation, may be a carrier of sphingosine 1-phosphate (S1P), which plays an essential role in chemoresistance [45, 46]. Additionally, Takafumi Tamura et al. reported that a low HDL-C level was related to a poor prognosis in gastric cancer [47]. However, we could not conclude that HDL-C is associated with clinical outcomes because only two studies were included. Therefore, additional studies are required.

Inflammation-based prognostic scoring systems considering inflammatory cells (lymphocytes, neutrophils, monocytes and platelets) and proteins (ALB, CRP and HDL-C) are useful because these factors play an important role in the proliferation and metastasis of tumor cells.

There are several highlights in this analysis. First, compared with previous studies based on inflammatory biomarkers in nasopharyngeal carcinoma, such as Li Su et al. [48], both inflammatory cells and proteins were considered in our study, and we included a larger sample size. In their study, Li Su et al indicated that lymphocyte counts and NLR may serve as prognostic makers, although only 14 studies involving 11,651 NPC patients were included. Second, our study included more valuable predicative biomarkers. Tomohiro F. Nishijima et al. [49] and Liangyou $\mathrm{Gu}$ et al. [50] demonstrated the prognostic significance of LMR in several malignancies; however, only 3 retrospective studies in these meta-analyses assessed the prognostic value of LMR on NPC. To date, several meta-analyses have indicated that an elevated NLR is a valuable predictive biomarker of a 
poor prognosis in patients with NPC [51,52], and the study performed by Yi Fang et al. also indicated that elevated serum CRP levels were related to a worse prognosis in NPC patients [53]. However, these meta-analyses only focused on one biomarker. Third, the biomarkers in our study are inexpensive and easily available. Although the prognostic value of Epstein-Barr virus DNA load and circulating microRNAs in NPC patients has been reported in a meta-analysis [54], in contrast to the hematological markers in our study, the detection of the Epstein-Barr virus DNA load and circulating microRNAs is expensive and poorly repeatable. Moreover, both random-effects and fixed-effects models were used in our meta-analysis, providing a more comprehensive understanding of the results. Nonetheless, there are also some limitations. First, except for one study, the eligible studies were all retrospective analyses. Second, because the cut-off values were identified based on various criteria, the cut-off values for the hematological parameters differed among the included studies. Third, the hazard ratios were obtained from univariate analyses if multivariate analyses were not available. Fourth, only articles published in English were included. Moreover, almost all eligible studies were conducted in the Chinese population, and only 2 studies were conducted in Singapore. Thus, the conclusions might be confined to the East Asian population because compared with other parts of the world, there are many more cases and a much higher incidence of NPC in China and Southeast Asia [55]. Overall, for a biomarker to be used in the clinic, it should be assessed in a systematic manner, and more clinical data are needed to confirm this conclusion.

\section{Conclusion}

This meta-analysis suggests that inflammatory cells (such as neutrophils, monocytes, lymphocytes and platelets) and proteins (such as ALB, CRP and HDL-C) have the capacity to predict survival in cancer, including NPC. As widely available and inexpensive biomarkers, hematological parameters may facilitate prognosis prediction in patients with solid tumors. However, prospective and multicenter studies on larger scales are needed to confirm our findings.

\section{Abbreviations}

HR: hazard ratio; NA: not available; NLR: neutrophil-to-lymphocyte ratio; PLR: platelet-tolymphocyte ratio; LMR: lymphocyte-to-monocyte ratio; CRP/ALB: C-reactive protein/albumin ratio; GPS: Glasgow prognostic score; HDL-C: high-density lipoprotein cholesterol; HNLR: high neutrophil-to- lymphocyte; LNLR: low neutrophil-to-lymphocyte ratio; h-HDL: high high-density lipoprotein cholesterol; 1-HDL: low high-density lipoprotein cholesterol; H-Fibrinogen: high fibrinogen; L-Fibrinogen: low fibrinogen; OS: overall survival; PFS: progression-free survival; DFS: disease-free survival; DMFS: distant metastasis-free survival.

\section{Competing Interests}

The authors have declared that no competing interest exists.

\section{References}

1. Wei KR, Zheng RS, Zhang SW, Liang ZH, Ou ZX, Chen WQ. Nasopharyngeal carcinoma incidence and mortality in China in 2010. Chinese journal of cancer. 2014; 33: 381-7.

2. Adham M, Kurniawan AN, Muhtadi AI, Roezin A, Hermani B, Gondhowiardjo S, et al. Nasopharyngeal carcinoma in Indonesia: epidemiology, incidence, signs, and symptoms at presentation. Chinese journal of cancer. 2012; 31: 185-96.

3. Parkin DM, Bray F, Ferlay J, Pisani P. Global cancer statistics, 2002. Ca-Cancer J Clin. 2005; 55: 74-108.

4. $\mathrm{Xu} \mathrm{C}$, Chen YP, Ma J. Clinical trials in nasopharyngeal carcinoma-past, present and future. Chinese clinical oncology. 2016; 5: 20

5. Lee AW, Lau WH, Tung SY, Chua DT, Chappell R, Xu L, et al. Preliminary results of a randomized study on therapeutic gain by concurrent chemotherapy for regionally-advanced nasopharyngeal carcinoma: NPC-9901 Trial by the Hong Kong Nasopharyngeal Cancer Study Group. Journal of clinical oncology : official journal of the American Society of Clinical Oncology. 2005; 23: 6966-75.

6. Sun R, Qiu HZ, Mai HQ, Zhang Q, Hong MH, Li YX, et al. Prognostic value and differences of the sixth and seventh editions of the UICC/AJCC staging systems in nasopharyngeal carcinoma. Journal of cancer research and clinical oncology. 2013; 139: 307-14.

7. Xu J, Wan XB, Huang XF, Chan KC, Hong MH, Wang LH, et al. Serologic antienzyme rate of Epstein-Barr virus DNase-specific neutralizing antibody segregates TNM classification in nasopharyngeal carcinoma. Journal of clinical oncology : official journal of the American Society of Clinical Oncology. 2010; 28: 5202-9.

8. Min GT, Wang YH, Yao N, Zhao JM, Wang J, Wang HP, et al. The prognostic role of pretreatment platelet-to-lymphocyte ratio as predictors in patients with colorectal cancer: a meta-analysis. Biomark Med. 2017; 11: 87-97.

9. Zhu YY, Si W, Sun Q, Qin BY, Zhao WH, Yang JL. Platelet-lymphocyte ratio acts as an indicator of poor prognosis in patients with breast cancer. Oncotarget. 2017; 8: 1023-30.

10. Kinoshita A, Onoda H, Imai N, Iwaku A, Oishi M, Tanaka K, et al. The C-reactive protein/albumin ratio, a novel inflammation-based prognostic score, predicts outcomes in patients with hepatocellular carcinoma. Annals of surgical oncology. 2015; 22: 803-10.

11. Gao Y, Huang D. The value of the systematic inflammation-based Glasgow Prognostic Score in patients with gastric cancer: a literature review. Journal of cancer research and therapeutics. 2014; 10: 799-804.

12. Nishijima TF, Muss HB, Shachar SS, Tamura K, Takamatsu Y. Prognostic value of lymphocyte-to-monocyte ratio in patients with solid tumors: A systematic review and meta-analysis. Cancer Treat Rev. 2015; 41: 971-8.

13. Gu L, Li H, Chen L, Ma X, Li X, Gao Y, et al. Prognostic role of lymphocyte to monocyte ratio for patients with cancer: evidence from a systematic review and meta-analysis. Oncotarget. 2016; 7: 31926-42.

14. Selzer E, Grah A, Heiduschka G, Kornek G, Thurnher D. Primary radiotherapy or postoperative radiotherapy in patients with head and neck cancer. Strahlentherapie Und Onkologie. 2015; 191: 486-94.

15. Moher D, Liberati A, Tetzlaff J, Altman DG, Group P. Preferred reporting items for systematic reviews and meta-analyses: the PRISMA statement. Journal of clinical epidemiology. 2009; 62: 1006-12.

16. Tierney JF, Stewart LA, Ghersi D, Burdett S, Sydes MR. Practical methods for incorporating summary time-to-event data into meta-analysis. Trials. 2007; 8: 16.

17. Chua M, Ong WS, Tan SH, Fong KW, Soong YL, Wee J, et al. EBV DNA and neutrophil-lymphocyte ratio as predictive biomarkers for induction chemotherapy in advanced nasopharyngeal carcinoma. Ann Oncol. 2015; 26: 99.

18. Lan M, Chen CY, Huang Y, Mao MJ, Han F, Liao JF, et al. Elevated plasma fibrinogen level shows superior prognostic value than Epstein-Barr virus DNA load for stage IVA/B nasopharyngeal carcinoma patients in the intensity-modulated radiotherapy era. Oncotarget. 2016; 7: 46242-52.

19. Gooden MJ, de Bock GH, Leffers N, Daemen T, Nijman HW. The prognostic influence of tumour-infiltrating lymphocytes in cancer: a systematic review with meta-analysis. British journal of cancer. 2011; 105: 93-103. 
20. Balermpas P, Rodel F, Weiss C, Rodel C, Fokas E. Tumor-infiltrating lymphocytes favor the response to chemoradiotherapy of head and neck cancer. Oncoimmunology. 2014; 3: e27403.

21. Zikos TA, Donnenberg AD, Landreneau RJ, Luketich JD, Donnenberg VS. Lung T-cell subset composition at the time of surgical resection is a prognostic indicator in non-small cell lung cancer. Cancer Immunol Immun. 2011; 60: 819-27.

22. Rosenberg SA. Progress in human tumour immunology and immunotherapy. Nature. 2001; 411: 380-4.

23. Chan OSH, Kowanetz M, Ng WT, Koeppen H, Chan LK, Yeung RMW, et al. Characterization of PD-L1 expression and immune cell infiltration in nasopharyngeal cancer. Oral Oncol. 2017; 67: 52-60.

24. Gay LJ, Felding-Habermann B. Contribution of platelets to tumour metastasis. Nature reviews Cancer. 2011; 11: 123-34

25. Sullivan LA, Brekken RA. The VEGF family in cancer and antibody-based strategies for their inhibition. Mabs-Austin. 2010; 2: 165-75.

26. Goubran HA, Burnouf T, Radosevic M, El-Ekiaby M. The platelet-cancer loop. Eur J Intern Med. 2013; 24: 393-400.

27. Peterson JE, Zurakowski D, Italiano JE, Michel LV, Connors S, Oenick M, et al. VEGF, PF4 and PDGF are elevated in platelets of colorectal cancer patients. Angiogenesis. 2012; 15: 265-73.

28. Ishizuka M, Nagata H, Takagi K, Iwasaki Y, Kubota K. Combination of platelet count and neutrophil to lymphocyte ratio is a useful predictor of postoperative survival in patients with colorectal cancer. British journal of cancer. 2013; 109: 401-7.

29. Rachidi S, Wallace K, Day TA, Alberg AJ, Li Z. Lower circulating platelet counts and antiplatelet therapy independently predict better outcomes in patients with head and neck squamous cell carcinoma. Journal of hematology \& oncology. 2014; 7: 65 .

30. Tecchio C, Scapini P, Pizzolo G, Cassatella MA. On the cytokines produced by human neutrophils in tumors. Seminars in cancer biology. 2013; 23: 159-70.

31. Leek RD, Harris AL. Tumor-associated macrophages in breast cancer. Journal of mammary gland biology and neoplasia. 2002; 7: 177-89.

32. Hutterer GC, Pichler M, Chromecki TF, Strini KA, Klatte T, Pummer K, et al. Tumour-associated macrophages might represent a favourable prognostic indicator in patients with papillary renal cell carcinoma. Histopathology. 2013; 63: 309-15.

33. Bingle L, Brown NJ, Lewis CE. The role of tumour-associated macrophages in tumour progression: implications for new anticancer therapies. The Journal of pathology. 2002; 196: 254-65.

34. Yim GW, Eoh KJ, Kim SW, Nam EJ, Kim YT. Malnutrition Identified by the Nutritional Risk Index and Poor Prognosis in Advanced Epithelial Ovarian Carcinoma. Nutrition and cancer. 2016; 68: 772-9.

35. Liu Z, Jin K, Guo M, Long J, Liu L, Liu C, et al. Prognostic Value of the CRP/Alb Ratio, a Novel Inflammation-Based Score in Pancreatic Cancer. Annals of surgical oncology. 2017; 24: 561-8.

36. Zhou T, Zhan JH, Hong SD, Hu ZH, Fang WF, Qin T, et al. Ratio of C-Reactive Protein/Albumin is An Inflammatory Prognostic Score for Predicting Overall Survival of Patients with Small-cell Lung Cancer. Sci Rep-Uk. 2015; 5.

37. Wei XL, Wang FH, Zhang DS, Qiu MZ, Ren C, Jin Y, et al. A novel inflammation-based prognostic score in esophageal squamous cell carcinoma: the C-reactive protein/albumin ratio. BMC Cancer. 2015; 15: 350.

38. Saijo M, Nakamura K, Masuyama H, Ida N, Haruma T, Kusumoto T, et al. Glasgow prognostic score is a prognosis predictor for patients with endometrial cancer. Eur J Obstet Gyn R B. 2017; 210: 355-9.

39. Pan QX, Su ZJ, Zhang JH, Wang CR, Ke SY. Glasgow Prognostic Score predicts prognosis of intrahepatic cholangiocarcinoma. Molecular and clinical oncology. 2017; 6: 566-74.

40. Omichi C, Nakamura K, Haraga J, Masuyama H, Hiramatsu Y. Glasgow prognostic score is an independent marker for poor prognosis with all cases of epithelial ovarian cancer. Cancer medicine. 2016; 5: 1074-80.

41. Martino MM, Briquez PS, Ranga A, Lutolf MP, Hubbell JA. Heparin-binding domain of fibrin(ogen) binds growth factors and promotes tissue repair when incorporated within a synthetic matrix. P Natl Acad Sci USA. 2013; 110: 4563-8.

42. Zhang X, Long Q. Elevated serum plasma fibrinogen is associated with advanced tumor stage and poor survival in hepatocellular carcinoma patients. Medicine. 2017; 96

43. Luo YL, Kim HS, Kim M, Lee M, Song YS. Elevated plasma fibrinogen levels and prognosis of epithelial ovarian cancer: a cohort study and meta-analysis. J Gynecol Oncol. 2017; 28

44. Zeng Q, Xue N, Dai D, Xing S, He X, Li S, et al. A Nomogram based on Inflammatory Factors C-Reactive Protein and Fibrinogen to Predict the Prognostic Value in Patients with Resected Non-Small Cell Lung Cancer. Journal of Cancer. 2017; 8: 744-53.

45. Colie S, Van Veldhoven PP, Kedjouar B, Bedia C, Albinet V, Sorli SC, et al. Disruption of Sphingosine 1-Phosphate Lyase Confers Resistance to Chemotherapy and Promotes Oncogenesis through Bcl-2/Bcl-xL Upregulation. Cancer Research. 2009; 69: 9346-53.

46. Sato K, Okajima F. Role of sphingosine 1-phosphate in anti-atherogenic actions of high-density lipoprotein. World journal of biological chemistry. 2010; 1 : 327-37.

47. Tamura T, Inagawa S, Hisakura K, Enomoto T, Ohkohchi N. Evaluation of serum high-density lipoprotein cholesterol levels as a prognostic factor in gastric cancer patients. J Gastroen Hepatol. 2012; 27: 1635-40.
48. Su L, Zhang MW, Zhang WJ, Cai CS, Hong JS. Pretreatment hematologic markers as prognostic factors in patients with nasopharyngeal carcinoma A systematic review and meta-analysis. Medicine. 2017; 96.

49. Nishijima TF, Muss HB, Shachar SS, Tamura K, Takamatsu Y. Prognostic value of lymphocyte-to-monocyte ratio in patients with solid tumors: A systematic review and meta-analysis. Cancer Treatment Reviews. 2015; 41: 971-8.

50. Gu LY, Li HZ, Chen LY, Ma X, Li XT, Gao Y, et al. Prognostic role of lymphocyte to monocyte ratio for patients with cancer: evidence from a systematic review and meta-analysis. Oncotarget. 2016; 7: 31926-42.

51. Mascarella MA, Mannard E, Silva SD, Zeitouni A. Neutrophil-to-lymphocyte ratio in head and neck cancer prognosis: A systematic review and meta-analysis. Head And Neck-Journal for the Sciences And Specialties Of the Head And Neck. 2018; 40: 1091-100.

52. Takenaka Y, Kitamura T, Oya R, Ashida N, Shimizu K, Takemura K, et al. Prognostic role of neutrophil-lymphocyte ratio in nasopharyngeal carcinoma: A meta-analysis. PloS one. 2017; 12: e0181478.

53. Fang $\mathrm{Y}, \mathrm{Xu} \mathrm{C}, \mathrm{Wu} \mathrm{P}$, Zhang $\mathrm{LH}$, Li DW, Sun JH, et al. Prognostic role of C-reactive protein in patients with nasopharyngeal carcinoma: A meta-analysis and literature review. Medicine. 2017; 96

54. Liu TB, Zheng ZH, Pan J, Pan LL, Chen LH. Prognostic role of plasma Epstein-Barr virus DNA load for nasopharyngeal carcinoma: a meta-analysis. Clin Invest Med. 2017; 40: E1-E12.

55. Petersson F. Nasopharyngeal carcinoma: a review. Seminars in diagnostic pathology. 2015; 32: 54-73.

56. Chen C, Sun P, Dai QS, Weng HW, Li HP, Ye S. The Glasgow Prognostic Score predicts poor survival in cisplatin-based treated patients with metastatic nasopharyngeal carcinoma. PloS one. 2014; 9: e112581.

57. He JR, Shen GP, Ren ZF, Qin H, Cui C, Zhang Y, et al. Pretreatment levels of peripheral neutrophils and lymphocytes as independent prognostic factors in patients with nasopharyngeal carcinoma. Head And Neck-Journal for the Sciences And Specialties Of the Head And Neck. 2012; 34: 1769-76.

58. Sun W, Zhang LL, Luo M, Hu GY, Mei Q, Liu DB, et al. Pretreatment hematologic markers as prognostic factors in patients with nasopharyngeal carcinoma: Neutrophil-lymphocyte ratio and platelet-lymphocyte ratio. Head and Neck-Journal for the Sciences and Specialties of the Head and Neck. 2016; 38: E1332-E40.

59. Jiang R, Cai XY, Yang ZH, Yan Y, Zou X, Guo L, et al. Elevated peripheral blood lymphocyte-to-monocyte ratio predicts a favorable prognosis in the patients with metastatic nasopharyngeal carcinoma. Chinese journal of cancer. 2015; 34: 237-46.

60. Jiang R, Zou X, Hu W, Fan YY, Yan Y, Zhang MX, et al. The elevated pretreatment platelet-to-lymphocyte ratio predicts poor outcome in nasopharyngeal carcinoma patients. Tumour biology : the journal of the International Society for Oncodevelopmental Biology and Medicine. 2015; 36: 7775-87.

61. Jin $Y, Y e \quad X$, He CX, Zhang BB, Zhang YP. Pretreatment neutrophil-to-lymphocyte ratio as predictor of survival for patients with metastatic nasopharyngeal carcinoma. Head and Neck-Journal for the Sciences and Specialties of the Head and Neck. 2015; 37: 69-75.

62. Li J, Jiang R, Liu WS, Liu Q, Xu M, Feng QS, et al. A large cohort study reveals the association of elevated peripheral blood lymphocyte-to-monocyte ratio with favorable prognosis in nasopharyngeal carcinoma. PloS one. 2013; 8: e83069.

63. Li XH, Chang $\mathrm{H}, \mathrm{Xu}$ BQ, Tao YL, Gao J, Chen C, et al. An inflammatory biomarker-based nomogram to predict prognosis of patients with nasopharyngeal carcinoma: an analysis of a prospective study. Cancer medicine. 2017; 6: 310-9.

64. Lin GN, Peng JW, Liu DY, Xiao JJ, Chen YQ, Chen XQ. Increased lymphocyte to monocyte ratio is associated with better prognosis in patients with newly diagnosed metastatic nasopharyngeal carcinoma receiving chemotherapy. Tumor Biology. 2014; 35: 10849-54.

65. Li JP, Chen SL, Liu XM, He X, Xing $S$, Liu YJ, et al. A Novel Inflammation-Based Stage (I Stage) Predicts Overall Survival of Patients with Nasopharyngeal Carcinoma. International journal of molecular sciences. 2016; 17

66. An $\mathrm{X}$, Ding PR, Wang FH, Jiang WQ, Li YH. Elevated neutrophil to lymphocyte ratio predicts poor prognosis in nasopharyngeal carcinoma. Tumour biology : the journal of the International Society for Oncodevelopmental Biology and Medicine. 2011; 32: 317-24.

67. Chang H, Gao J, Xu BQ, Guo SP, Lu RB, Li G, et al. Haemoglobin, neutrophil to lymphocyte ratio and platelet count improve prognosis prediction of the TNM staging system in nasopharyngeal carcinoma: development and validation in 3,237 patients from a single institution. Clinical oncology (Royal College of Radiologists (Great Britain)). 2013; 25: 639-46

68. Chua MLK, Tan SH, Kusumawidjaja G, Shwe MTT, Cheah SL, Fong KW, et al. Neutrophil-to-lymphocyte ratio as a prognostic marker in locally advanced nasopharyngeal carcinoma: A pooled analysis of two randomised controlled trials. European Journal of Cancer. 2016; 67: 119-29.

69. Chua M, Ong WS, Tan SH, Fong KW, Soong YL, Wee J, et al. EBV DNA and neutrophil-lymphocyte ratio as predictive biomarkers for induction chemotherapy in advanced nasopharyngeal carcinoma. Annals of Oncology. 2015; 26: ix99.

70. Lu AY, Li HF, Zheng YM, Tang MZ, Li J, Wu HH, et al. Prognostic Significance of Neutrophil to Lymphocyte Ratio, Lymphocyte to Monocyte Ratio, and 
Platelet to Lymphocyte Ratio in Patients with Nasopharyngeal Carcinoma. Biomed Res Int. 2017.

71. Tao CJ, Chen $\mathrm{YY}$, Jiang F, Feng $\mathrm{XL}$, Jin $\mathrm{QF}$, Jin $\mathrm{T}$, et al. The C-reactive Protein/Albumin Ratio Is an independent Prognostic Factor for Overall Survival in Patients with Nasopharyngeal Carcinoma Receiving Intensity-Modulated Radiotherapy. Journal of Cancer. 2016; 7: 2005-11.

72. He SS, Wang Y, Chen HY, Yang L, Liang SB, Lu LX, et al. C-Reactive Protein/Albumin Ratio (CAR) as a Prognostic Factor in Patients with Non-Metastatic Nasopharyngeal Carcinoma. Journal of Cancer. 2016; 7: 2360-6.

73. Zhang Y, Zhou GQ, Liu X, Chen L, Li WF, Tang LL, et al. Exploration and Validation of C-Reactive Protein/Albumin Ratio as a Novel Inflammation-Based Prognostic Marker in Nasopharyngeal Carcinoma. Journal of Cancer. 2016; 7: 1406-12.

74. Liu YY, Lin SJ, Chen YY, Liu LN, Bao LB, Tang LQ, et al. High-density lipoprotein cholesterol as a predictor of poor survival in patients with nasopharyngeal carcinoma. Oncotarget. 2016; 7: 42978-87.

75. Jiang R, Yang ZH, Luo DH, Guo L, Sun R, Chen QY, et al. Elevated apolipoprotein A-I levels are associated with favorable prognosis in metastatic nasopharyngeal carcinoma. Med Oncol. 2014; 31: 80.

76. He SS, Wang Y, Yang L, Chen HY, Liang SB, Lu LX, et al. Plasma Fibrinogen Correlates with Metastasis and is Associated with Prognosis in Human Nasopharyngeal Carcinoma. Journal of Cancer. 2017; 8: 403-9.

77. Tang LQ, Chen QY, Guo SS, Chen WH, Li CF, Zhang L, et al. The impact of plasma epstein-barr virus DNA and fibrinogen on nasopharyngeal carcinoma prognosis: an observational study. British journal of cancer. 2014; 111: 1102-11. 